\title{
Singular sector of the Burgers-Hopf hierarchy and deformations of hyperelliptic curves
}

\author{
Yuji Kodama and Boris G. Konopelchenko
}

\begin{abstract}
We discuss the structure of shock singularities of the Burgers-Hopf hierarchy. It is shown that the set of singular solutions defines a stratification of the affine space of the flow parameters in the hierarchy. The stratification is associated with the Birkhoff decomposition of the Grassmannian given by the set of linear spaces spanned by the hierarchy. We then construct integrable hierarchy on each stratum and demonstrate that it describes a deformation of a hyperelliptic curve parametrizing the stratum. The hierarchy is called the hidden Burgers-Hopf hierarchy, and we found the Riemann invarint form and the hodograph solution.
\end{abstract}

\section{Introduction: Singular solutions of the BH hierarchy}

The Burgers-Hopf (BH) hierarchy defined by

$$
\frac{\partial u}{\partial t_{n}}=c_{n} u^{n} \frac{\partial u}{\partial x}, \quad \text { with } \quad c_{n}=(-1)^{n} \frac{(2 n+1) ! !}{2^{n} n !}, \quad n=0,1,2, \cdots,
$$

plays an important role in the study of a wide range of phenomena in physics from hydrodynamics to topological field theory (for examples, see [23, 7, 8]). It is clear that the $\mathrm{BH}$ hierarchy can be obtained by the dispersionless limit of the $\mathrm{KdV}$ hierarchy as well as the dissipationless limit of the Burgers hierarchy. However the present study does not require any information of the original hierarchy.

Using the method of characteristic, the solution of the hierarchy can be expressed by the hodograph form with $t_{0} \equiv x$,

$$
\Omega\left(u, x, t_{1}, t_{2}, \cdots\right):=x+\sum_{j=1}^{\infty} c_{j} u^{j} t_{j}-f(u)=0,
$$

where $f(u)$ may be determined by the initial data, e.g. $u(x, 0,0, \cdots)=f^{-1}(x)$ for a monotone data. Then the BH hierarchy gives a deformation of the data $x=f(u)$ in each $t_{j}$-direction, and this defines an infinite dimensional surface given by (1.1).

Here we are interested in the class of solutions of the BH hierarchy with singularity of shock wave type. The singularity of this type corresponds to a point on the surface where the derivative $\partial u / \partial x$ blows up at this point, that is, the equation (1.1) is not invertible for $u$. This condition is equivalent to $\Omega^{\prime}:=\partial \Omega / \partial u=0$ at the point, which implies that the solution $u$ can be expressed as a function of

1991 Mathematics Subject Classification. Primary 37K10 58F07; Secondary 35Q53 14H20.

Key words and phrases. integrable systems, singularity theory. 
$\left(t_{1}, t_{2}, \cdots\right)$. Thus the conditions $\Omega=0$ and $\Omega^{\prime}=0$ define a singular sector of codimension $\geq 1$ in $\mathbb{C}^{\infty}$. The affine space $\mathbb{C}^{\infty}$ is then stratified with those singular sectors as follows: Let us first defined the solution sets,

$$
U_{n}:=\left\{u \mid \Omega^{(j)}=0(0 \leq j \leq n), \Omega^{(n+1)} \neq 0\right\},
$$

where $\Omega^{(i)}=\partial^{i} \Omega / \partial u^{i}$. Then the singular sector of codimension $m$ can be defined as

$$
Z_{m}:=\left\{\left(t_{0}, t_{1}, t_{2}, \cdots\right) \in \mathbb{C}^{\infty} \mid u \in U_{j} \text { such that } m=\sum_{j=1}^{\infty} j\left|U_{j}\right| .\right\} .
$$

where $\left|U_{j}\right|$ is the number of solutions in $U_{j}$. For examples, $Z_{0}$ consists of the points where $\Omega(u)=0$ has only simple roots, and the points in $Z_{1}$ correspond to the case where $\Omega(u)=0$ and $\Omega^{\prime}(u)=0$ has just only one common root and no roots for $\Omega^{(j)}(u)=0, j \geq 2$. The closure of the set of those singular sectors then forms a decomposition of the affine space $\mathbb{C}^{\infty}$, i.e.

$$
\mathbb{C}^{\infty}=\overline{\bigcup_{m \geq 0} Z_{m}}, \quad \text { disjoint union. }
$$

In order to show some explicit structure of the set $Z_{m}$, let us consider the case where $f(u)$ is given by a $(N+1)$ th degree of polynomial in $u$. Then on a $(N+1)$ dimensional subspace of $\mathbb{C}^{\infty}$ with $t_{n}=0$ for $n \geq N+1$, the equation (1.1) can be written by

$$
\Omega_{N+1}\left(u, x, t_{1}, \cdots, t_{N}\right)=x+\sum_{j=1}^{N} c_{j} u^{j} t_{j}=u^{N+1}+\sum_{j=0}^{N} b_{j} u^{j} .
$$

Since all the coefficients $b_{j}$ 's on the right hand side can be absorbed by shifting the times, $\left(x-b_{0}, t_{1}-\left(b_{1} / c_{1}\right), t_{2}-\left(b_{2} / c_{2}\right), \cdots\right)$, we just set all $b_{j}=0$. Then we consider the singular sectors, denoted as $Z_{m}^{N+1}$, on the subspace $\mathbb{C}^{N+1}$. The equation 1.3 ) is well known as a universal unfolding of a singularity of $A_{N}$-type [5. The singularity of this type includes cusp, swallow tail and butterfly for $N=2,3$ and 4 . As an example we just consider the case with $N=2$.

EXAMPLE 1.1. The hodograph solution (1.3) with $N=2$ is given by

$$
\Omega_{3}\left(u, x, t_{1}, t_{2}\right)=x-\frac{3}{2} u t_{1}+\frac{15}{8} u^{2} t_{2}-u^{3}=0 .
$$

Then the singular sector $Z_{1}^{3}$ is given by

$$
Z_{1}^{3}=Z_{1}^{3+} \cup Z_{1}^{3-}, \quad \text { with } \quad Z_{1}^{3 \pm}=\left\{\left(x, t_{1}, t_{2}\right) \mid \Omega_{3}\left(u^{ \pm}, x, t_{1}, t_{2}\right)=0, u^{+} \neq u^{-}\right\},
$$

where $u^{ \pm}$are the roots of $\Omega_{3}^{\prime}=0$, i.e.

$$
u^{ \pm}=\frac{1}{8}\left(5 t_{2} \pm \sqrt{25 t_{2}^{2}-32 t_{1}}\right) .
$$

The sets $Z_{1}^{3 \pm}$ obviously intersects on the double points of $u^{+}=u^{-}$, and forms a cusp singularity. The intersection provides the higher singular sector $Z_{2}^{3}$ which is given by a twisted cubic curve,

$$
Z_{2}^{3}=\left\{\left(x, t_{1}, t_{2}\right) \mid x=u^{3}, t_{1}=2 u^{2}, t_{2}=(8 / 5) u\right\} .
$$


The real sections of those sectors will be an interesting object to study.

REMARK 1.2. A stratification of certain (compact) manifold is an interesting problem of algebraic geometry, and has several intimate connections with the study of integrable systems. For examples, the isospectral manifolds of the periodic Toda lattices are given by Jacobian varieties which have the stratification based on the theta divisor [2, 1, 15]. Also in the case of finite nonperiodic Toda lattices, the isospectral manifolds are compactified in the flag manifolds, and are decomposed into the Bruhat cells which correspond to the sets of singular solutions [11, 的. (See also [22] for several examples of the stratifications of Jacobian varieties related to finite dimensional hamiltonian systems.) The present study will provide an example of the stratification related to integrable systems of hydrodynamic type.

In this paper, we construct an integrable hierarchy defined on each singular sector $Z_{m}$. Those hierarchies can be also obtained by the dispersionless limits of the hidden hierarchies of the KdV equation considered in 19. We call those the hidden $\mathrm{BH}$ hierarchies, and we construct those as a regularization of the shock singularity of the $\mathrm{BH}$ hierarchy. Each hierarchy on $Z_{m}$ is called the $\mathrm{BH}_{m}$ hierarchy. In Section 2, we discuss the Grassmannian structure of the singular sectors and show the connection to hyperelliptic curves. Then in Section 3, we define the $\mathrm{BH}_{m}$ hierarchy on $Z_{m}$ as a deformation of a hyperelliptic curve of genus $m$. We also show that the hierarchy can be put into the Riemann invariant form, and then construct the hodograph solution. The Riemann invariant form has been also obtained in a different approach in 9]. In Section 4, we give a brief discussion on several extensions of the present construction of the hidden dispersionless hierarchies. We also mention some related topics such as a topological field theory [4] and a possible stratification of the Frobenius manifold as obtained by those hidden hierarchies 14.

\section{Grassmannian structure of the BH hierarchy}

In this section we discuss the Grassmannian structure of the solutions of the $\mathrm{BH}$ hierarchy. Let us first recall (see for example [13]) that the BH hierarchy can be formulated as

$$
\frac{\partial p}{\partial t_{j}}=\frac{\partial}{\partial x} Q_{j}, \quad \text { with } \quad Q_{j}=\left(\lambda^{2 j+1}\right)_{+p}, j=0,1,2, \cdots,
$$

where $\left(\lambda^{n}\right)_{+p}$ indicates the polynomial part of $\lambda^{n}$ in $p$ having the algebraic relation,

$$
p^{2}=\lambda^{2}+u \text {. }
$$

The hierarchy (2.1) can be considered as a deformation of the curve (2.2) which corresponds to the Riemann surface of genus 0 (sphere) with the compactification. Later we will use $k=\lambda^{2}$, and then we assign the degrees as

$$
\operatorname{Deg}(k)=2, \quad \operatorname{Deg}(p)=1 .
$$

One introduces a function $S\left(x, t_{1}, t_{2}, \cdots\right)$ which plays an important role in the theory of dispersionless hierarchy. The $S$ function is defined by rewriting (2.1) in the form,

$$
\frac{\partial S}{\partial t_{j}}=Q_{j}, \quad \text { for } \quad j=0,1,2, \cdots
$$


Since $Q_{j}=\left(\lambda^{2 j+1}\right)_{+p}$ is a polynomial of degree $2 j+1$ in $p$ and $p^{2}=k+u, k=\lambda^{2}$, the $S$ function can be expressed by a Laurent series in $\mathbb{C}\left[\left[k, k^{-1}\right]\right] \cdot \sqrt{k}$,

$$
S\left(t_{0}, t_{1}, t_{2}, \cdots\right)=\sum_{i=0}^{\infty} k^{i+\frac{1}{2}} t_{i}+\sum_{j=0}^{\infty} \frac{1}{k^{j+\frac{1}{2}}} F_{j}\left(t_{0}, t_{1}, t_{2}, \cdots\right) .
$$

To characterize the solution space of the BH hierarchy, we now define a linear space spanned by the flows (2.3) in a manner similar to the case of the KP hierarchy [3],

$$
\begin{aligned}
W_{0} & :=\operatorname{Span}_{\mathbb{C}}\left\{S_{t_{0}}, S_{t_{1}}, S_{t_{2}}, \cdots\right\} \\
& =\operatorname{Span}_{\mathbb{C}}\left\{Q_{0}, Q_{1}, Q_{2}, \cdots\right\}
\end{aligned}
$$

Since $Q_{i}$ is a polynomial in $p$ and $p^{2}=k+u$, one can also consider $Q_{i}$ be an element in $\mathbb{C}[k] \cdot p$. This observation will be crucial for defining an integrable hierarchy on the singular sector. Thus we have

$$
W_{0}=\operatorname{Span}_{\mathbb{C}}\left\{p, k p, k^{2} p, \cdots\right\} \cong \mathbb{C}[k] \cdot p .
$$

This implies the inclusion,

$$
k W_{0} \subset W_{0},
$$

which is the condition for the KdV reduction in terms of the KP hierarchy [21].

One should however note that the linear space $W_{0}$ cannot be defined on the singular sectors where the flows (2.1) blow up and the commutativity among the $S$ flows is not defined. Recall that the conditions on the hodograph solution $\Omega=0$ provide a constraint on the coordinates. For example, the first condition $\Omega^{\prime}=0$ gives

$$
c_{1} t_{1}+\sum_{j=2}^{\infty} j c_{j} u^{j-1} t_{j}=f^{\prime}(u)
$$

which implies that $u$ can be determined as a function of $t_{j}$ for $j \geq 1$. Then with $\Omega=0$, the coordinate $x$ can be considered as a function of the other times. Hence one can expect to have a map $\psi^{m}: \mathbb{C}^{\infty} \longrightarrow Z_{m}$ so that

$$
\psi^{m}\left(t_{m}, t_{m+1}, t_{m+2}, \cdots\right)=\left(a_{0}, a_{1}, \cdots, a_{m-1}, t_{m}, t_{m+1}, \cdots\right)
$$

where $a_{i}, 0 \leq i \leq m-1$ are some functions of $t_{j}, j \geq m$. The map can be defined at least locally on the sector $Z_{m}$, and it defines a patch on $Z_{m}$. The $S$-function then takes the form,

$$
S^{m}\left(t_{m}, t_{m+1}, \cdots\right)=k^{m+\frac{1}{2}} \sum_{i=0}^{\infty} k^{i} t_{m+i}+\sum_{i=0}^{m-1} a_{i}\left(t_{m}, t_{m+1}, \cdots\right) k^{i+\frac{1}{2}}+O\left(k^{-\frac{1}{2}}\right) .
$$

The linear space spanned by the flows $\partial S^{m} / \partial t_{j} j \geq m$ under this restriction on the times defines

$$
W_{m}=\operatorname{Span}_{\mathbb{C}}\left\{S_{t_{m}}^{m}, S_{t_{m+1}}^{m}, S_{t_{m+2}}^{m} \cdots\right\} \cong k^{m} W_{0} .
$$

Then introducing new variable $p_{m}:=S_{t_{m}}^{m}$, we see $W_{m}$ as the polynomial ring,

$$
W_{m} \cong \mathbb{C}[k] \cdot p_{m}, \quad \text { as a vector space. }
$$

The Grassmannian $G r$ is a set of all linear spaces $W_{m}, m \geq 0$, and it has the Birkhoff decomposition [21,

$$
G r=\bigcup_{m \geq 0} \Sigma_{m}
$$


where the Birkhoff stratum $\Sigma_{m}$ is the set of all linear spaces of $W_{m}$. The structure of the Grassmannian is then the same as the KdV hierarchy by making the identification with $k=\lambda^{2}$,

$$
W_{m} \cong\left\{\lambda^{-m}, \lambda^{-m+2}, \cdots, \lambda^{m-2}, \lambda^{m}, \lambda^{m+1}, \cdots\right\},
$$

where $\lambda^{2} W_{m} \subset W_{m}$ (i.e. $k W_{m} \subset W_{m}$ ) [3]. The BH hierarchy is thus defined on the principal stratum $\Sigma_{0}$.

Since the degrees of $k$ and $p_{m}$ are assigned as

$$
\operatorname{Deg}(k)=2, \quad \operatorname{Deg}\left(p_{m}\right)=2 m+1,
$$

one can consider a plane curve $\mathcal{C}_{m}$ defined by

$$
\mathcal{C}_{m}:=\left\{\left(k, p_{m}\right) \in \mathbb{C}^{2} \mid p_{m}^{2}=k^{2 m+1}+\sum_{i=0}^{2 m} u_{i} k^{2 m-i}\right\},
$$

which defines a hyperelliptic curve of genus $m$ and is a smooth affine variety for the generic values of $u_{i}$ 's, the deformation parameters. Then the ring $W_{m}=\mathbb{C}[k] \cdot p_{m}$ can be considered as a part of the quotient ring $\mathbb{C}[k, p] / \mathcal{C}_{m}$ with the curve $\mathcal{C}_{m}$. One should also note that there is a natural reduction of the curve,

$$
\mathcal{C}_{m} \longrightarrow \mathcal{C}_{m-1} \quad\left(p_{m}=k p_{m-1}\right),
$$

which corresponds to the conditions on the parameters, $u_{2 m}=u_{2 m-1}=0$. This can be considered as a dispersionless analog of the regularization of the singularity in the KP hierarchy by the Bäcklund transformation [3]. Thus the stratum $\Sigma_{m}$ is parametrized by the curve $\mathcal{C}_{m}$, and the boundary of the stratum corresponds to a singular curve of degenerate genus $m$. The higher order stratum is obtained by the desingularization of the curve by increasing the genus. The curve $\mathcal{C}_{m}$ can be obtained by the dispersionless limit of the hidden $\mathrm{KdV}$ hierarchy considered in 19 and also by a quasi-classical $\bar{\partial}$ dressing method introduced in 18. In the next section we will consider a deformation of the curve by defining a system of equations for the parameters $u_{i}$ in $\mathcal{C}_{m}$ where the deformation is parametrized by the times $t_{j}$ for $j \geq m$.

\section{The hidden $\mathrm{BH}$ hierarchies}

We now construct an integrable deformation of the curve $\mathcal{C}_{m}$ in (2.6), which is defined on the singular sector $Z_{m}$. First recall the quotient ring of $\mathbb{C}\left[k, p_{m}\right]$ over an ideal $\mathcal{C}_{m}$ which has a split,

$$
\mathcal{R}_{m}:=\frac{\mathbb{C}\left[k, p_{m}\right]}{\mathcal{C}_{m}}=\mathbb{C}[k] \oplus \mathbb{C}[k] \cdot p_{m} .
$$

Then a deformation of the curve $\mathcal{C}_{m}$ with an infinite number of deformation parameters is defined in the same form as (2.3),

$$
\frac{\partial S^{m}}{\partial t_{j}}=Q_{j}^{m}, \quad Q_{j}^{m} \in \mathbb{C}[k] \cdot p_{m}, \quad j=0,1,2, \cdots,
$$

where $S^{m}$ is given by (2.4), and $\operatorname{Deg}\left(Q_{j}^{m}\right)=2(m+j)+1$ with $Q_{0}^{m}=p_{m}$. With this definition, the functions $a_{i}$ 's in $S^{m}$ can be explicitely written in terms of $u_{i}$ 's in the curve $\mathcal{C}_{m}$. Here we have relabeled the times as $t_{m+i} \rightarrow t_{i}$, but we do not think it will cause any confusion. As we will show below, all the flows in (3.1) 
are compatible for appropriate form of $Q_{j}^{m}$, that is, $\partial Q_{i}^{m} / \partial t_{j}=\partial Q_{j}^{m} / \partial t_{i}$, and in particular we have for the case $i=0$,

$$
\frac{\partial p_{m}}{\partial t_{j}}=\frac{\partial}{\partial x} Q_{j}^{m}
$$

where we denote $x=t_{0}$. This provides the system of equations of hydrodynamic type for $u_{i}$. Also notice that the flows (3.2) are compatible with the automorphism of the curve, $p_{m} \rightarrow-p_{m}$.

Now we show the compatibility of the flows in (3.1). We first note

LEMMA 3.1. The $Q_{i}^{m}$ 's in (3.1) are given by

$$
Q_{i}^{m}=\left(\frac{k^{m+i+\frac{1}{2}}}{p_{m}}\right)_{+} p_{m}, \quad \text { for } \quad i=0,1,2, \cdots,
$$

where $(\cdot)_{+}$denotes the projection onto $\mathbb{C}[k]$.

Proof. Since $\partial p_{m} / \partial t_{i} \sim O\left(k^{m-\frac{1}{2}}\right)$, one can set

$$
Q_{i}^{m} \sim k^{m+i+\frac{1}{2}}+O\left(k^{m-\frac{1}{2}}\right)
$$

This implies

$$
\frac{Q_{i}^{m}}{p_{m}} \sim \frac{k^{m+i+\frac{1}{2}}}{p_{m}}+O\left(k^{-1}\right) .
$$

The statement of the Lemma then follows from $Q_{i}^{m} / p_{m} \in \mathbb{C}[k]$.

This formula is a non-zero genus extension of the case with $m=0$. In fact we have

Corollary 3.1.

$$
Q_{i}=\left(k^{i+\frac{1}{2}}\right)_{+p}=\left(\frac{k^{i+\frac{1}{2}}}{p}\right)_{+} p, \quad \text { with } \quad p^{2}=k+u,
$$

where $Q_{i}$ is defined in (2.1) with $k=\lambda^{2}$.

Proof. We first note

$$
\left(\frac{k^{i+\frac{1}{2}}}{p}\right)_{+} p=k^{i+\frac{1}{2}}-\left(\frac{k^{i+\frac{1}{2}}}{p}\right)_{-} p,
$$

where $(.)_{-}$is the non-polynomial part in $k$. Since $\operatorname{Deg}(k)=2$ and $\operatorname{Deg}(p)=1$, we have

$$
\left(\frac{k^{i+\frac{1}{2}}}{p}\right)_{-} p=\left(\left(\frac{k^{i+\frac{1}{2}}}{p}\right)_{-} p\right)_{-p}=\left(k^{i+\frac{1}{2}}\right)_{-p} .
$$

This implies the result.

In order to show the compatibility of the flows (3.1), we note

LEMMA 3.2. The flows (3.1) can be put into the Lax form,

$$
\frac{\partial k}{\partial t_{i}}=\left\{Q_{i}^{m}, k\right\}:=\frac{\partial Q_{i}^{m}}{\partial p_{m}} \frac{\partial k}{\partial x}-\frac{\partial Q_{i}^{m}}{\partial x} \frac{\partial k}{\partial p_{m}},
$$

where $p_{m}$ is then considered to be a constant parameter. 
Proof. The flows $\partial p_{m} / \partial t_{i}=\partial Q_{i}^{m} / \partial x$ in (3.1) with $x=t_{0}$ can be written in terms of the differential three forms,

$$
d p \wedge d k \wedge d x=d Q_{i}^{m} \wedge d t_{i} \wedge d k .
$$

Then assuming $k=k(p, x, t)$, we obtain the result.

It is standard that the following Proposition proves the compatibility of the flows, that is, $\partial^{2} k / \partial t_{i} \partial t_{j}=\partial^{2} k / \partial t_{j} \partial t_{i}$ :

Proposition 3.1.

$$
\frac{\partial Q_{i}^{m}}{\partial t_{j}}-\frac{\partial Q_{j}^{m}}{\partial t_{i}}+\left\{Q_{i}^{m}, Q_{j}^{m}\right\}=0 .
$$

Proof. Note that writing $Q_{i}^{m}=\beta_{i}^{+} p_{m}$ with $\beta_{i}^{+} \in \mathbb{C}[k]$, the equation (3.4) can be expressed by the following equation for $\beta_{i}^{+}$'s,

where

$$
\frac{\partial \beta_{i}^{+}}{\partial t_{j}}-\frac{\partial \beta_{j}^{+}}{\partial t_{i}}+\left\{\left\{\beta_{i}^{+}, \beta_{j}^{+}\right\}\right\}=0
$$

$$
\{\{f, g\}\}:=f \frac{\partial g}{\partial x}-g \frac{\partial f}{\partial x}+p_{m}\{f, g\}
$$

This equation can be proven as follows: First note

$$
\frac{\partial \beta_{i}}{\partial t_{j}}=\left\{\left\{\beta_{j}^{+}, \beta_{i}\right\}\right\}, \quad \text { for } \quad \beta_{i}=\frac{k^{m+i+\frac{1}{2}}}{p_{m}}, \quad\left(\beta_{j}^{+}=\left(\beta_{j}\right)_{+}\right) .
$$

With the decomposition $\beta_{i}=\beta_{i}^{+}+\beta_{i}^{-}$, we have

$$
\frac{\partial \beta_{i}^{+}}{\partial t_{j}}+\frac{\partial \beta_{i}^{-}}{\partial t_{j}}=\left\{\left\{\beta_{j}^{+}, \beta_{i}^{+}\right\}\right\}+\left\{\left\{\beta_{j}^{+}, \beta_{i}^{-}\right\}\right\}
$$

We also have

$$
\frac{\partial \beta_{j}^{+}}{\partial t_{i}}+\frac{\partial \beta_{j}^{-}}{\partial t_{i}}=-\left\{\left\{\beta_{i}^{-}, \beta_{j}^{+}\right\}\right\}-\left\{\left\{\beta_{i}^{-}, \beta_{j}^{-}\right\}\right\}
$$

where we have used $\left\{\left\{\beta_{i}, \beta_{j}\right\}\right\}=0$. Combining those two equations and projecting on $\mathbb{C}[k]$, we obtain the compatibility equation for $\beta_{i}^{+}$as well as $\beta_{i}^{-}$. This completes the proof. $\square$

The simplest example of the $\mathrm{BH}_{m}$ equations in terms of $u_{i}$ 's is given by the $t_{1}$-flow of the $\mathrm{BH}_{1}$ equation (see Remark 3.2 below for the general form),

$$
\frac{\partial}{\partial t_{1}}\left(\begin{array}{l}
u_{0} \\
u_{1} \\
u_{2}
\end{array}\right)=\left(\begin{array}{ccc}
-\frac{3}{2} u_{0} & 1 & 0 \\
-u_{1} & -\frac{1}{2} u_{0} & 1 \\
-u_{2} & 0 & -\frac{1}{2} u_{0}
\end{array}\right) \frac{\partial}{\partial x}\left(\begin{array}{l}
u_{0} \\
u_{1} \\
u_{2}
\end{array}\right) .
$$

As a consequence of the Lax form (3.3), we have

COROLlary 3.2. The $\mathrm{BH}_{m}$ hierarchy (3.3) can be put into the Riemann invariant form,

$$
\frac{\partial \kappa_{i}}{\partial t_{j}}=\phi_{i}^{j} \frac{\partial \kappa_{i}}{\partial x}, \quad \text { for } \quad i=0,1,2, \cdots, 2 m
$$

where the Riemann invariants $\kappa_{i}$ 's are the roots of the polynomial associated with the curve $\mathcal{C}_{m}$, and $\phi_{i}^{j}$ is given by the $\left(\partial Q_{j}^{m} / \partial p_{m}\right)(k)$ at $k=\kappa_{i}$, that is,

$$
p_{m}^{2}=\prod_{i=0}^{2 m}\left(k-\kappa_{i}\right), \quad \text { and } \quad \phi_{i}^{j}=\phi^{j}\left(\kappa_{i}\right)=\left.\left(\frac{k^{j+m+\frac{1}{2}}}{p}\right)_{+}\right|_{k=\kappa_{i}} .
$$


Proof. First note that from the curve $p_{m}^{2}=F(k)$ we have

$$
2 p_{m}=\frac{\partial F}{\partial k} \cdot \frac{\partial k}{\partial p_{m}}
$$

from which $\partial k / \partial p_{m}=0$ implies $p_{m}=0$. Then the evaluation of $(3.3)$ at the roots of $p_{m}^{2}=0$ completes the proof. $\square$

For example, the Riemann invariant form for the first flow of the $\mathrm{BH}_{m}$ equation is given by

$$
\frac{\partial \kappa_{i}}{\partial t_{1}}=\left(\kappa_{i}-\frac{1}{2} u_{0}\right) \frac{\partial \kappa_{i}}{\partial x}, \quad i=0,1, \cdots, 2 m
$$

where $u_{0}=-\sum_{i=0}^{2 m} \kappa_{i}$. The Riemann invariant form has been obtained in [9]. We here note that the Riemann invariant form has an obvious reduction with $\kappa_{j}=0$ for some $j$ 's. This corresponds to the reduction of the genus as mentioned in the end of the previous section. There is also another reduction with all $\kappa_{0}=\cdots=\kappa_{2 m}$ which is associated to a degenerate case of $\mathcal{C}_{m}$ equivalent to $p_{m}^{2}=k^{2 m+1}$ of genus 0 .

With the form (3.5), we can construct the hodograph solution of the hierarchy as shown in 13. We first remark that the functions $\phi^{j}(k)$ in 3.5$)$ form a finite ring of dimension $2 m+1$,

$$
\mathcal{F}_{m}:=\frac{\mathbb{C}[k]}{p_{m}^{2}}=\operatorname{Span}_{\mathbb{C}}\left\{\phi^{0}, \phi^{1}, \cdots, \phi^{2 m}\right\} .
$$

Note here that $\phi^{j}(k)$ is a monic polynomial of $\operatorname{Deg}\left(\phi^{j}\right)=2 j$ with $\operatorname{Deg}(k)=2$ and $\phi^{0}=1$. Then one can write the higher $\phi^{2 m+j}$ for $j \geq 1$ in the form,

$$
\phi^{2 m+j}=\sum_{i=0}^{2 m} \mu_{i}^{j}(\kappa) \phi^{i} \bmod p_{m}^{2}
$$

where $\mu_{i}^{j}(\kappa)$ are functions of the roots $\kappa_{i}$ of $p_{m}^{2}=0$. We also note that the $\mu_{i}^{j}(\kappa)$ 's are functions of the symmetric polynomials $\nu_{i}$ of the roots $\kappa_{j}$, which are given by $\nu_{i}=(-1)^{i} u_{i-1}, i=1, \cdots, 2 m+1$ with

$$
\nu_{1}=\sum_{j=0}^{2 m} \kappa_{j}, \quad \nu_{2}=\sum_{i<j} \kappa_{i} \kappa_{j}, \quad \nu_{3}=\sum_{i<j<l} \kappa_{i} \kappa_{j} \kappa_{l} \quad \cdots \quad \nu_{2 m+1}=\prod_{i=0}^{2 m} \kappa_{i} .
$$

For example, we have for $j=1$

$$
\mu_{2 m}^{1}=-\frac{3}{2} u_{0}, \quad \mu_{2 m-1}^{1}=-\frac{3}{2} u_{1}-\frac{3}{8} u_{0}^{2}, \quad \mu_{2 m-2}^{1}=-\frac{3}{2} u_{2}-\frac{3}{4} u_{0} u_{1}+\frac{1}{16} u_{0}^{3}, \cdots .
$$

Then we have

$$
\frac{\partial \kappa_{l}}{\partial t_{2 m+j}}=\sum_{i=0}^{2 m} \mu_{i}^{j}(\kappa) \frac{\partial \kappa_{l}}{\partial t_{i}}, \quad l=0,1, \cdots, 2 m
$$

which implies that all the roots $\kappa_{l}$ are constants along the characteristic curve,

$$
\frac{d t_{0}}{\mu_{0}^{j}(\kappa)}=\cdots=\frac{d t_{2 m}}{\mu_{2 m}^{j}(\kappa)}=\frac{d t_{2 m+j}}{-1}, \quad \text { for all } j \geq 1 \text {. }
$$

The integration of this equation gives

$$
t_{i}^{0}=t_{i}+\sum_{j=1}^{\infty} \mu_{i}^{j}(\kappa) t_{2 m+j}, \quad i=0,1, \cdots, 2 m
$$


where $t_{i}^{0}$ is the initial position of the characteristic at $t_{2 m+j}=0, j \geq 1$. As a simplest example with $m=1$, we have an explicit solution from (3.7) by setting $t_{2 m+j}=(3 / 2) \delta_{j 1}$ and $t_{i}^{0}=0$,

$$
u_{0}=t_{2}, \quad u_{1}=t_{1}-\frac{1}{4} t_{2}^{2}, \quad u_{2}=x-\frac{1}{2} t_{1} t_{2}+\frac{1}{6} t_{2}^{3} .
$$

Also taking the sum $\sum_{i=0}^{2 m} \phi^{i} t_{i}^{0}$ of (3.7), we obtain

THEOREM 3.1. The hodograph solution of the $\mathrm{BH}_{m}$ hierarchy is given by

$$
x+\sum_{i=0}^{\infty} \phi^{i}\left(\kappa_{j}\right) t_{i}=0, \quad j=0,1, \cdots, 2 m,
$$

where the times $\left(x, t_{1}, t_{2}, \cdots\right)$ can be shifted arbitrary.

This formula will be useful to study the singular structure of the $\mathrm{BH}_{m}$ hierarchy as in the case of the BH hierarchy. We will report a detailed analysis elsewhere.

REMARK 3.2. The general form of the $\mathrm{BH}_{m}$ hierarchy can be written in the following form with the polynomial $\phi^{i}(k)$ for $k$ being replaced by a matrix $K$,

$$
\frac{\partial U}{\partial t_{i}}=\phi^{i}(K) \frac{\partial U}{\partial x}, \quad \text { with } \quad U=\left(u_{0}, u_{1}, \cdots, u_{2 m}\right)^{T},
$$

where $K$ is the (companion) matrix given by

$$
K=\left(\begin{array}{ccccc}
-u_{0} & 1 & \cdots & \cdots & 0 \\
-u_{1} & 0 & \cdots & \cdots & 0 \\
\vdots & \vdots & \ddots & \ddots & \vdots \\
-u_{2 m-1} & 0 & \cdots & \cdots & 1 \\
-u_{2 m} & 0 & \cdots & \cdots & 0
\end{array}\right)
$$

For example, $\phi^{1}(K)=K-(1 / 2) u_{0} I$ with $I=(2 m+1) \times(2 m+1)$ identity matrix.

\section{Further extensions and discussion}

The present construction of the hidden hierarchies can be extended to the case with the hyperelliptic curve associated with an even degree polynomial,

$$
\tilde{\mathcal{C}}_{m}:=\left\{(k, p) \in \mathbb{C}^{2} \mid p^{2}=k^{2 m+2}+\sum_{i=1}^{2 m+2} v_{i} k^{2 m+2-i}\right\} .
$$

Here the degree of $k$ is assigned as $\operatorname{Deg}(k)=1$ so that $\operatorname{Deg}(p)=m+1$. The hierarchy of deformations of the curve $\tilde{\mathcal{C}}_{m}$ can be also obtained as the dispersionless limit of the integrable hierarchy associated with the energy-dependent Schrödinger potentials considered in 20], which coincides with the hidden hierarchy for the Jaulent-Miodek hierarchy [12 (see also [17]). We here call the integrable hierarchy associated with the curve $\mathcal{\mathcal { C }}_{m}$ the $\mathrm{dJM}_{m}$ hierarchy. Most of the results obtained in Sections 2 and 3 remains the same for this case. In particular, the singular sectors $Z_{m}$ for the $\mathrm{dJM}_{0}$ hierarchy are given by the higher order intersections of two curves given by the hodograph solution for $v_{1}$ and $v_{2}$, which are defined as follows: Let the curve $\tilde{\mathcal{C}}_{0}$ be given by

$$
p^{2}=k^{2}+v_{1} k+v_{2}=\left(k-\kappa_{1}\right)\left(k-\kappa_{2}\right) .
$$


The hodograph solution has the same form as (1.1),

$$
\Omega^{j}:=x+\sum_{i=0}^{\infty} \phi^{i}\left(\kappa_{j}\right) t_{i}=0, \quad j=1,2 .
$$

Then the singular sectors are defined by

$$
Z_{m}=\left\{\left(t_{0}, t_{1}, t_{2}, \cdots\right) \in \mathbb{C}^{\infty}\left|\left(\kappa_{1}, \kappa_{2}\right) \in U_{j}, m=\sum_{j=1}^{\infty} j\right| U_{j} \mid\right\},
$$

where $U_{j}$ are defined by

$$
U_{j}=\left\{\left(\kappa_{1}, \kappa_{2}\right) \mid \Omega^{1}=\Omega^{2}=0, \tilde{\Omega}_{(i)}=0(1 \leq i \leq j), \tilde{\Omega}_{(j+1)} \neq 0\right\} .
$$

Here the function $\tilde{\Omega}_{(i)}\left(\kappa_{1}, \kappa_{2}\right)$ may be given by $\tilde{\Omega}_{(i)}=\left.D\right|_{\omega=\Omega_{2}^{1} / \Omega_{1}^{1}}$ with,

$$
D:=\left|\begin{array}{cc}
\left(\Omega_{1}^{1}\right)^{i} & \left(\Omega_{1}^{2}\right)^{i} \\
\Omega_{(i)}^{1} & \Omega_{(i)}^{2}
\end{array}\right|, \quad \Omega_{(i)}^{j}=\left(\frac{\partial}{\partial \kappa_{2}}-\omega\left(\kappa_{1}, \kappa_{2}\right) \frac{\partial}{\partial \kappa_{1}}\right)^{i} \Omega^{j}
$$

where $\Omega_{j}^{i}=\partial \Omega^{i} / \partial \kappa_{j}$. Note that $\tilde{\Omega}_{(1)}$ is just the Jacobian determinant. The singular structure of the dispersionless JM hiearchy is then similar to the case of the $\mathrm{BH}$ hierarchy. We will report a detailed discussion on the stratification determined by the $\mathrm{dJM}_{m}$ hierarchies elsewhere.

One should also note that the integrable deformation of the algebraic curve defined by either $\mathrm{BH}_{m}$ or $\mathrm{dJM}_{m}$ hierarchy is rather a general property of the dispersionless integrable hierarchy. For example, the dispersionless limit of the first hidden KP hierarchy discussed in [16] leads to the standard form of the elliptic curve,

$$
\mathcal{C}_{K P}: p^{2}+v_{0} p q+v_{1} p=q^{3}+u_{0} q^{2}+u_{1} q+u_{2},
$$

and the corresponding deformation has the form,

$$
\left\{\begin{array}{l}
\frac{\partial p}{\partial t_{n}}=\frac{\partial}{\partial y} Q_{n}, \\
\frac{\partial q}{\partial t_{n}}=\frac{\partial}{\partial x} Q_{n},
\end{array} \quad \text { with } \quad Q_{n} \in \frac{\mathbb{C}[p, q]}{\mathcal{C}_{K P}}, \quad n=0,1,2, \cdots,\right.
$$

where $\operatorname{Deg}\left(Q_{n}\right)=n+2$ with $\operatorname{Deg}(p)=3, \operatorname{Deg}(q)=2$ and $t_{0}=x, t_{1}=y$. This contains the $\mathrm{BH}_{1}$ hierarchy as the reduction with $q=k=$ constant and $v_{0}=v_{1}=0$, and the hidden dispersioless Boussinesq hierarchy with the reduction $p=k=$ constant and $u_{0}=0$. The singular structures determined by the hidden dispersionless KP hierarchy and its reductions (such as the Gel'fand-Dikii type) will be discussed elsewhere. We also remark that the integrable deformation of hyperelliptic curve discussed in the present paper is different from that given by the Whitham hierarchy (see for example [10). The Whitham hierarchy describes a slow modulation over a quasi-periodic solution of the original dispersive equation, and is obtained as a dispersive regularization of the dispersionless hierarchy. However our deformation describes each stratum (singular sector) of the Birkhoff decomposition, which is parametrized by the hyperelliptic curve, and it has no direct connection with the original dispersive equation.

As a final remark, we would like to point out that the $\mathrm{BH}_{m}$ hierarchy may not have a single free energy (or prepotential) which plays a central role of a topological field theory. In the dispersionless KP hierarchy, the free energy can be obtained by 
integrating twice the function $G_{i j}$ (referred as the Gel'fand-Dikii potential in [4]) defined as the flux density of the conservation laws,

$$
\frac{\partial g_{j}}{\partial t_{i}}=\frac{\partial}{\partial x} G_{i j}
$$

Here $g_{j}=G_{0 j}$ and $G_{i j}$ are the coefficients of the expansions of $p_{m}$ and $Q_{i}^{m}$ in (3.2). In the case of the $\mathrm{BH}_{m}$, they are given by the residue formulae,

$$
G_{i j}=\operatorname{Res}_{k=\infty}\left(Q_{i}^{m} k^{j-m} \frac{d k}{\sqrt{k}}\right) .
$$

Because of the conservation law (4.1), $G_{i j}$ can be integrated once to get the form with some function $F_{j}$,

$$
G_{i j}=\frac{\partial}{\partial t_{i}} F_{j}
$$

(Note that $F_{j}$ are related with the functions $a_{i}$ 's in (2.4) as $F_{j}=a_{m-1-j}$ for $0 \leq j \leq m-1$.) However one can show that $G_{i j}$ is not a symmetric function with respect to the indices $i, j$ except the case with $m=0$. Hence there is no further integration to express the $G_{i j}$ as a two point function as in the case of dispersionless KP theory (see for example [4, 14]), such as,

$$
G_{i j}=\left\langle\phi^{i} \phi^{j}\right\rangle=\frac{\partial^{2}}{\partial t_{i} \partial t_{j}} F
$$

where $\phi_{i}$ 's are the primary fields or graviational decendants and $F$ represents the free energy of the topological field theory. Thus the $\mathrm{BH}_{m}$ hierarchy is somewhat different from most of the known dispersionless hierarchies which have a direct connection to topological field theory [7, 4]. It is then quite interesting to construct a topological field theory based on the $\mathrm{BH}_{m}$ hierarchies, and if there exists such a theory, then the associated Frobenius manifold may have a stratified structure characterized by a finite ring related to $\mathcal{F}_{m}$ in (3.6).

Aknowledgement: YK is supported in part by NSF Grant \#DMS0071523. BGK is supported in part by the grant COFIN 2000 "Sintesi", and is grateful to Department of Mathematics, Ohio State University for the kind hospitality and support. BGK would like to thank L. Martinez Alonso, A. Mikhailov and A. Veselov for useful discussions.

Note added in proof: After the completion of this paper, M. Pavlov informed us that the $\mathrm{BH}_{m}$ hierarchy can be transformed into the Whitham equation correspoding to the $m$-gap solution of the KdV equation by a reciprocal transformation. We thank M. Pavlov for this information.

\section{References}

[1] M. Adler, L. Haine and P. van Moerbeke, Limit matrices for the Toda flow and periodic flags for loop groups, Math. Ann. 296 (1993) 1-33.

[2] M. Adler and P. van Moerbeke, The Toda lattice, Dynkin diagrams, singularities and Abelian varieties, Invent. Math. 103 (1991) 223-278.

[3] M. Adler and P. van Moerbeke, Birkhoff strata, bäcklund transformations and regularization of isospectral operators, Adv. Math. 108 (1994) 140-204.

[4] S. Aoyama and Y. Kodama, Topological Landau-Ginzburg theory with a rational potential and the dispersionless KP theory, Comm. Math. Phys., 182 (1996) 185-219. 
[5] V. I. Arnol'd, V. A. Vasil'ev, V. V. Goryunov and O. V. Lyashko, Singularities Local and Global Teory, in Encyclopaedia of Mathematical Sciences: Dynamical Systems VI, (SpringerVerlag, Berlin, 1993).

[6] L. Casian and Y. Kodama, Blow ups of the Toda lattices and their intersections with the Bruhat cells, (preprint, 2002) to appear in Contemporary Math.

[7] R. Dijkgraaf and E. Witten, Mean field theory, topological field theory and multi-matrix models, Nucl. Phys., B342 (1990) 486-522.

[8] B. Dubrovin and Y. Zhang, Bi-Hamiltonian hierarchy in 2D topological field theory at oneloop approximation, Comm. Math. Phys., 198 (1998) 311-361.

[9] E. V. Ferapontov and M. V. Pavlov, Quasiclassical limit of coupled KdV equations, Riemann invariants and multi-Hamiltonian structure, Physica D, 52 (1991) 211-219.

[10] H. Flaschka, M. G. Forest and D. W. McLaughlin, Multiphase averaging and the inverse spectral solution of the Korteweg-de Vries equation, Comm. Pure Appl. Math., 33 (1980) 739-784.

[11] H. Flaschka and L. Haine. Varietes de drapeaux et reseaux de Toda. Mathematische Zeitschrift, 208 (1991), 545-556.

[12] M. Jaulent and Y. Miodek, Nonlinear evolution equations associated with "energy-dependent Schrödinger potentials", Lett. Math. Phys. 1 (1975/77) 243-250

[13] Y. Kodama and J. Gibbons, A method for solving the dispersionless KP hierarchy and its exact solutions II, Phys. Lett. 135 (1989) 167-170.

[14] Y. Kodama, Dispersionless integrable systems and their solutions, in Integrability: SeibergWitten and Whitham equations (Edinburgh, 1998) Eds. H. W. Branden and I. M. Krichever (Gordon and Breach, Amsterdam, 2000) 199-212.

[15] Y. Kodama, Topology of the real part of hyperelliptic Jacobian associated with the periodic Toda lattice, nlin.SI/0201009).

[16] B. G. Konopelchenko, L. Martinez Alonso and E. Medina, Singular sector of the KadomtsevPetviashvili hierarchy, $\bar{\partial}$ operators of nonzero index, and associated integrable systems, $J$. Math. Phys. 41 (2000) 385-413.

[17] B. G. Konopelchenko, L. Martinez Alonso and E. Medina, Hidden integrable hierarchies of AKNS type, J. Phys. A: Math. Gen. 32 (1999) 3621-3635.

[18] B. G. Konopelchenko, L. Martinez Alonso and O. Ragnisco, The $\bar{\partial}$-approach to the dispersionless KP hierarchy, J. Phys. A: Math. Gen., 34 (2001) 10209-10217.

[19] M. Manas, L. Martinez Alonso and E. Medina, Zero sets of $\tau$-functions and hidden hierarchies of KdV type. J. Phys. A: Math. Gen. 30 (1997) 4815-4824.

[20] L. Martinez Alonso, Schrödinger spectral probles with energy-dependent potentials as sources of nonlinear Hamiltonian evolution equations, J. Math. Phys., 21 (1980) 2342-2349.

[21] A. Pressley and G. Segal, Loop groups, (Clarendon Press, Oxford, 1986).

[22] P. Vanhaecke, Integrable systems in the realm of algebraic geometry, Lecture Notes in Mathematics, 2nd Ed. 1638 (Springer-Verlag, Berlin, 2001).

[23] G. Whitham, Linear and Nonlinear Waves, (Wiley-Interscience, 1974).

Department of Mathematics, Ohio State University, Columbus, OH 43210, USA

E-mail address: kodama@math.ohio-state.edu

Dipartimento di Fisica, Universita di Lecce and Sezione INFN, 73100 Lecce, Italy

E-mail address: konopel@le.infn.it 